Rev. Biol. Neotrop. 4(2): 101-110. 2007

\title{
ISTAgem florística das Cyperaceae hidRófilas da Região do alto e médio rio Araguaia, Goiás, Mato Grosso e Tocan- tins, Brasil - Parte I
}

\author{
André dos Santos Bragança Gil \\ Programa de Pós-Graduação em Biologia Vegetal, Instituto de Biologia, Departamento de \\ Botânica, Universidade Estadual de Campinas, Barão Geraldo, 13083-970, Campinas, São \\ Paulo, Brasil; e-mail: asbgil@yahoo.com.br
}

\section{Adriana Luiza Ribeiro de Oliveira Claudia Petean Bove}

Museu Nacional, Departamento de Botânica, Universidade Federal do Rio de Janeiro, Quinta da Boa Vista, São Cristóvão, 20940-040, Rio de Janeiro, Rio de Janeiro, Brasil

\begin{abstract}
Resumo: Este trabalho apresenta a primeira parte da listagem florística das Cyperaceae hidrófilas ocorrentes na região do alto e médio rio Araguaia, localizada entre os estados de Goiás, Mato Grosso e Tocantins, no Brasil. A identificação dos táxons foi obtida com auxílio de literatura especializada e consulta às coleções dos herbários UEC, UFG, R e RB. Até o momento, foram identificados 10 gêneros e 29 espécies de Cyperaceae para a área em estudo. São fornecidos dados sobre a distribuição geográfica e a lista de material examinado das espécies analisadas.
\end{abstract}

Palavras-chave: Cyperaceae, Eleocharis, hidrófilas, rio Araguaia.

Aвstract: This work provides the first part of the checklist of hydrophilous Cyperaceae from the upper and middle Araguaia river, located in the states of Goiás, Mato Grosso and Tocantins, in Brazil. The taxa were identified based on the literature and by comparison with the following herbarium collections: UEC, UFG, R and RB. So far, 10 genera and 29 species have been identified for the studied area. The geographic distribution and a list of the specimens examined are presented.

KeY words: Cyperaceae, Eleocharis, Hydrophilous, Araguaia river.

\section{INTRODUÇÃO}<smiles>O</smiles>

Brasil possui a maior rede hidrográfica do mundo, representando o maior suprimento hídrico da América do Sul (Tundisi \& Barbosa, 1995), o que confere ao país grande riqueza e diversidade de ecossistemas aquáticos e, conseqüentemente, rica flora hidrófila.

A flora associada aos ecossistemas aquáticos compreende não apenas as plantas que vivem estritamente na água, como também espécies anfíbias que periodicamente encontram-se submersas, emergentes ou margeiam estes ambientes (Bove et al., 2003). Os vegetais hidrófilos apresentam grande capacidade de adaptação e extensa amplitude ecológica, pos- sibilitando que a mesma espécie colonize os mais diferentes tipos de ambientes (Esteves, 1988).

O rio Araguaia, principal afluente do rio Tocantins, está dividido em três grandes regiões (alto, médio e baixo Araguaia), segundo divisão didática apresentada pelo Departamento Nacional de Produção Viária Naval na década de 1980. A região do alto rio Araguaia, com $450 \mathrm{~km}$ de extensão, compreende desde as nascentes até a cidade de Registro do Araguaia, Goiás. O médio rio Araguaia, com $1.505 \mathrm{~km}$ de extensão, tem início em Registro do Araguaia e segue até a localidade de Santa 
Isabel do Araguaia, Pará. A região do baixo rio Araguaia, com $160 \mathrm{~km}$ de extensão, vai de Santa Isabel do Araguaia até a confluência com o rio Tocantins (IBGE, 1989). A área como um todo está localizada na depressão Araguaia-Tocantins, abrangendo os estados de Mato Grosso, Goiás, Pará e Tocantins, sendo vastamente irrigada pelos afluentes do rio Araguaia (Figura 1).

A bacia do Araguaia configura-se como uma região de grande diversidade florística, apresentando imensas áreas pouco conhecidas, temporária ou permanentemente alagadas e ricas em vegetação hidrófila.

A acelerada ação antrópica, decorrente principalmente da expansão das fronteiras agropecuárias que atinge a Região CentroOeste, vem ameaçando e, muitas vezes, destruindo esses ecossistemas.

O primeiro estudo envolvendo as hidrófitas brasileiras foi publicado por Hoehne (1948), que destacou a importância dos gêneros Cyperus L., Eleocharis R. BR. e Rhynchospora Vahl nos ambientes aquáticos da Região Centro-Oeste. Dubs (1998) apresentou uma listagem das fanerógamas terrestres e aquáticas ocorrentes nos estados de Mato Grosso e Mato Grosso do Sul, realizando um extenso levantamento em diversos herbários nacionais e internacionais, no qual relaciona 133 espécies de ciperáceas.

A família Cyperaceae é composta por cerca de 5.000 espécies distribuídas em duas subfamílias, 12 tribos e 112 gêneros (Bruhl, 1995). A diversidade de gêneros e espécies nesta família varia de acordo com os autores. Goetghebeur (1998) reconhece quatro subfamílias, 14 tribos e 104 gêneros. Judd et al. (2002) citam a ocorrência de 104 gêneros e 4.500 espécies. Soltis et al. (2005) creditam às Cyperaceae um número menor de gêneros (98) e de espécies (4.350).

É uma família com distribuição geográfica cosmopolita, que normalmente, mas não exclusivamente, ocorre em locais úmidos (Goetghebeur, 1998; Judd et al., 2002; Soltis et al., 2005). Possui grande destaque na vegetação pelo grande número de espécies ocorrentes em diversas regiões, caracterizando, inclusive, os ecossistemas aquáticos (Gil \& Bove, 2004). Os maiores gêneros de Cyperaceae são: Carex L. (2.000 spp.), Cyperus L. (600 spp.), Fimbristylis
Vahl (300 spp.), Scirpus L. (300 spp.), Rhynchospora (200 spp.), Scleria Berg. (200 spp.) e Eleocharis (200 spp.) (Judd et al., 2002).

Para o Brasil, Muniz \& Shepherd (1987) relataram 500 espécies de Cyperaceae e Luceño et al. (1997) apontaram a ocorrência de 600 a 700 espécies distribuídas em 40 a 44 gêneros.

Apesar de sua grande representatividade no país, as Cyperaceae têm sido pouco estudadas. Existem duas principais publicações que tratam desta família no Brasil: Nees (1842), para todo o país, e Barros (1960), apenas para o estado de Santa Catarina. Listagens, estudos taxonômicos e catálogos florísticos regionais são publicados com maior freqüência. Entre estes, destacam-se a listagem de Prata (2002) para o estado de Roraima, o estudo taxonômico de Gil \& Bove $(2004,2007)$ com Eleocharis do estado do Rio de Janeiro e o catálogo de Luceño et al. (1997) para Paraíba e Pernambuco. Existem, ainda, trabalhos com alguns grupos taxonômicos, entre os quais se destacam as contribuições ao gênero Rhynchospora (Guaglianone, 2001; Guaglianone \& Moore, 2001; Rocha \& Luceño, 2002; Thomas, 1996), a monografia do gênero Eleocharis (Svenson, $1929,1932,1934,1937,1939)$ e floras de maior amplitude, que tratam de espécies que ocorrem no Brasil, como a Flora Mesoamericana (Adams, 1994) e a Flora da Guiana Venezuelana (Kerns et al., 1998).

Apesar da importância da bacia do Araguaia no contexto mundial em que hoje se inserem todos os ecossistemas aquáticos, existem poucos trabalhos publicados sobre a flora da região, principalmente a vegetação hidrófila (Koehler \& Bove, 2001, 2004). Essa carência, aliada ao intenso impacto que esses ambientes vêm sofrendo, evidencia a urgente necessidade de estudos a respeito de sua vegetação, possibilitando não só o conhecimento acerca desta, como também a prática de ações conservacionistas.

O presente trabalho tem por objetivo contribuir para o conhecimento das espécies hidrófilas de Cyperaceae ocorrentes na região da bacia do rio Araguaia, fornecendo dados sobre os táxons levantados, sua distribuição geográfica e ocorrência a fim de implementar os estudos sistemáticos com a família nesta região. 


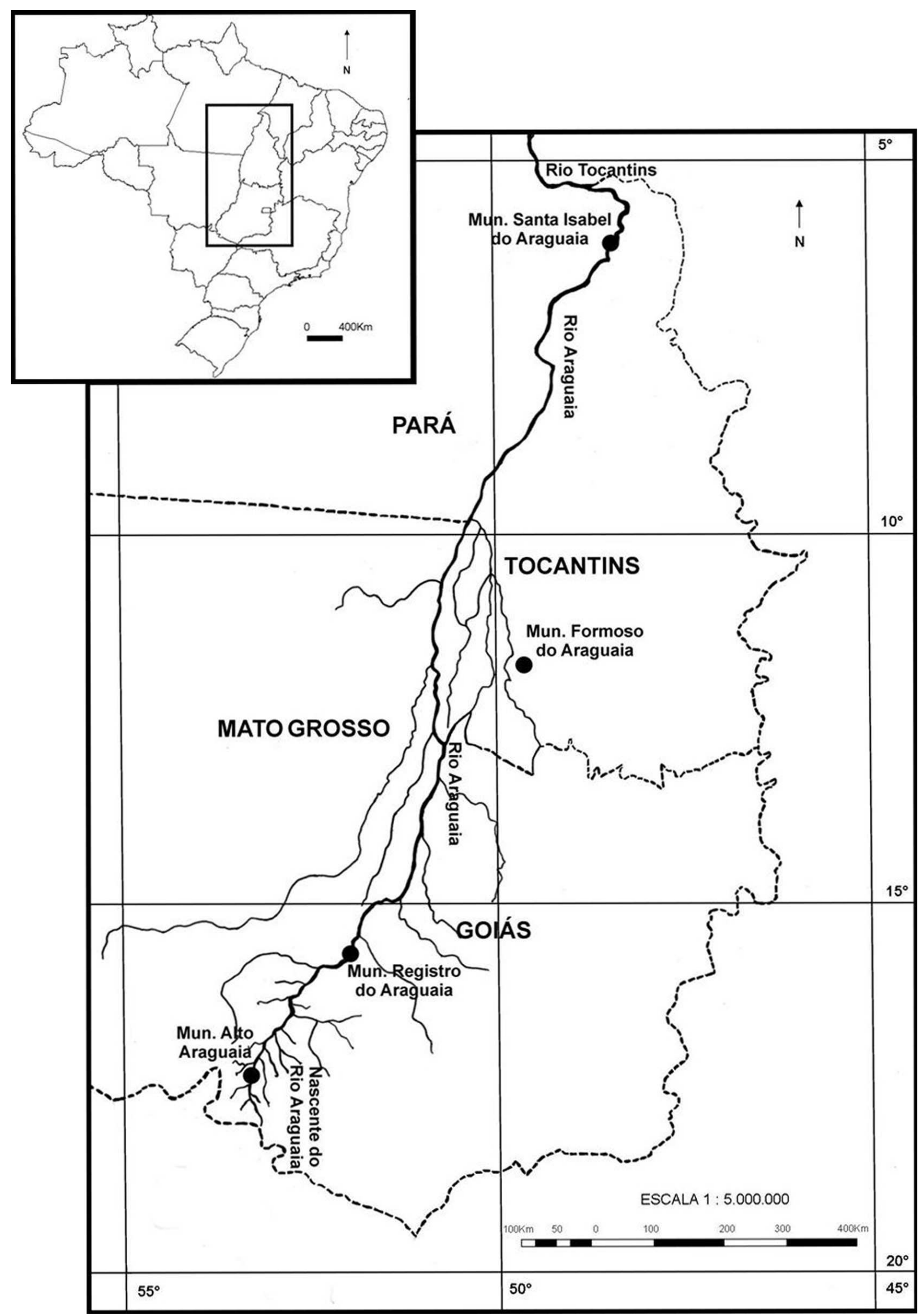

Figura 1 - Mapa descritivo da área estudada. 


\section{Material e métodos}

O material botânico analisado provém de coletas realizadas na região do alto e médio rio Araguaia, situada entre os paralelos $5^{\circ}-20^{\circ}$ de latitude Sul e $45^{\circ}-55^{\circ}$ de longitude Oeste. A área de coleta abrange uma vasta região, sendo o ponto extremo sul a cidade de Alto Araguaia, Mato Grosso e o ponto extremo norte, a cidade de Formoso do Araguaia, Tocantins (Figura 1). Entre estas localidades, foram realizadas expedições de coleta percorrendo as rodovias estaduais (MT-100, MT-126, MT-326, GO-164, GO-173, GO-221, GO-324, GO-334 e GO-530) e federais (BR-070, BR-158 e BR-251) em busca de ambientes aquáticos. As expedições foram realizadas nos períodos de maiojunho e outubro de 1997, novembro de 1999, janeiro de 2004 e abril de 2006, desta forma abrangendo períodos de chuva e de seca.

Também foram analisados materiais provenientes dos herbários UEC, UFG, $\mathrm{R}$ e RB. Os materiais depositados nos herbários HTO, NX e UB terão seus resultados publicados na Parte II desta listagem, pois ainda se encontram em estudo.

Procurou-se coletar material fértil que possuísse inflorescências jovens e maduras, verificando, in situ, características estruturais e ambientais. O material coletado foi preparado segundo técnicas tradicionais (Mori et al., 1989) e depositado no herbário do Museu Nacional do Rio de Janeiro (R).

Os nomes científicos e os basiônimos das espécies identificadas estão de acordo com os dados disponíveis no site do Missouri Botanical Garden (Tropicos.org. Missouri Botanical Garden, 2007). A identificação do material examinado foi obtida com auxílio de literatura específica e consulta às coleções dos herbários referidos anteriormente. Os dados de distribuição geográfica das espécies foram obtidos por intermédio da literatura (Adams, 1994; Barros, 1960; Diego-Pérez, 1997; Dubs, 1998; Faria, 1998; Gil \& Bove, 2007; Guaglianone, 2001; Guaglianone \& Moore, 2001; Hoehne, 1948; Irgang \& Gastal Jr., 1996; Jørgensen \& Ulloa, 1994; Kerns et al., 1998; Kuhlmann \& Kühn, 1947; Lawesson et al., 1987; Luceño et al., 1997; Marticorena \& Quezada, 1985; Martins et al., 1999; Matias et al. 2003; Muenscher, 1944; Muniz, 2001; Nees, 1842; Nelson, 1978;
Oliveira, 1980; Pedralli et al., 1993; Pott \& Pott, 1997; Prata, 2002; Renner et al., 1990; Rosa \& Irgang, 1998; Sampaio, 1916; Schessl, 1999; Simpson, 1995; Standley \& Steyermark, 1958; Svenson, 1929, 1932, 1934, 1937, 1939; Trevisan, 2005; Velasquez, 1994) e complementados com dados disponíveis no site do Missouri Botanical Garden (Tropicos.org. Missouri Botanical Garden, 2007).

\section{Resultados}

Até o momento, foi constatada a presença de 29 espécies distribuídas em dez gêneros para a área em estudo. Eleocharis mostrou-se o gênero mais representativo, perfazendo $34,5 \%$ do total de espécies identificadas.

1. Ascolepis brasiliensis (Kunth) Benth. ex. C. B. Clarke, Consp. Fl. Afr. 5: 651. 1894.

Platylepis brasiliensis Kunth, Enum. P1. 2: 269. 1837.

Material examinado: BRASIL, Goiás: Estrada Jussara-Britânia (via Jacilândia), a 78,2 km de Jussara, 12.XI.1999, C. P. Bove et al. 548 (R); Palestina, GO-221, km 52, 11.IV.2006, C. P. Bove et al. 1640 (R).

Distribuição geográfica: Panamá a Argentina, África Tropical e Madagascar. No Brasil, a espécie ocorre nos estados de Bahia, Goiás, Mato Grosso do Sul, Minas Gerais, Rio de Janeiro, Rio Grande do Sul, Roraima, Santa Catarina, São Paulo e no Distrito Federal.

2. Calyptrocarya glomerulata (Brongn.) Urb., Symb. Antill. 2: 169. 1900.

Becquerelia glomerulata Brongn., Voy. Monde 11: 163.1833.

Material examinado: BRASIL, Goiás: Cachoeira Abóbora, a $30 \mathrm{~km}$ de Caiapônia (sentido Piranhas), 18.XI.1999, C. P. Bove et al. 650 (R).

Distribuição geográfica: México, América Central, Caribe e América do Sul. No Brasil, a espécie ocorre nos estados de Acre, Amazonas, Bahia, Goiás, Mato Grosso, Mato Grosso do Sul, Pará, Paraíba, Pernambuco, Roraima e São Paulo.

3. Cyperus digitatus Roxb., Fl. Ind. ed.1: 209. 1820.

Material examinado: BRASIL, Goiás: Estrada para Peixe (GO-334), a 39,1 km da GO164, 16.XI.1999, C. P. Bove et al. 627 (R). 
Distribuição geográfica: sul dos Estados Unidos, México, América Central, Antilhas, América do Sul, Ásia, África e Madagascar. No Brasil, a espécie ocorre nos estados do Amazonas, Bahia, Goiás e Pernambuco.

4. Cyperus haspan L., Sp. Pl. 1: 45. 1753.

Material examinado: BRASIL, Goiás: 20 $\mathrm{km}$ ao sul de Caiapônia, na estrada para Jataí, 31.X.1964, H. S. Irwin E T. R. Soderstrom 7608 (UEC); estrada Jussara-Britânia (via Jacilândia), a 78,2 km de Jussara, 12.XI.1999, C. P. Bove et al. 538 (R); estrada Jussara-Aragarças, a $3 \mathrm{~km}$ de Jussara, Rio Água-Limpa, 11.XI.1999, C. P. Bove et al. 484 (R); estrada Jussara-Aragarças, a $100 \mathrm{~km}$ de Jussara, Rio Don Bill, 11.XI.1999, C. P. Bove et al. 517 (R); estrada Jussara-Aragarças, a $100 \mathrm{~km}$ de Jussara, Rio Don Bill, 11.XI.1999, C. P. Bove et al. 520 (R).

Distribuição geográfica: sul dos Estados Unidos, México, América Central, América do Sul, Ásia, África e Madagascar. No Brasil, ocorre nos estados de Bahia, Ceará, Espírito Santo, Goiás, Mato Grosso, Mato Grosso do Sul, Minas Gerais, Pará, Paraíba, Paraná, Pernambuco, Rio de Janeiro, Rio Grande do Sul, Roraima, Santa Catarina, São Paulo, Tocantins e no Distrito Federal. 1753.

5. Cyperus odoratus L., Sp. Pl. 1: 46.

Material examinado: BRASIL, Goiás: estrada Jussara-Aragarças, a $3 \mathrm{~km}$ de Jussara, Rio Água-Limpa, 11.XI.1999, C. P. Bove et al. 482 (R); estrada Jussara-Aragarças, a $111 \mathrm{~km}$ de Jussara, 11.XI.1999, C. P. Bove et al. 529 (R).

Distribuição geográfica: América do Norte, América Central, Caribe, Antilhas e América do Sul. No Brasil, a espécie ocorre nos estados do Acre, Bahia, Goiás, Mato Grosso, Minas Gerais, Paraíba, Pernambuco, Rio Grande do Sul e São Paulo.

6. Eleocharis acutangula (Roxb.) Schult., Mant. 2: 91. 1824.

Scirpus acutangulus Roxb., Fl. Ind. ed.1: 216. 1820.

Material examinado: BRASIL, Goiás: estrada Jussara-Aragarças, no km 100 (lado esquerdo), 26.V.1997, C. P. Bove et al. 186 (R); estrada Jussara-Aragarças, a $100 \mathrm{~km}$ de Jussara, 09.X.1997, C. P. Bove et al. 229 (R); estrada Aruanã-Britânia, no km 20, 14.X.1997, C. P. Bove et al. 290 (R); estrada Jussara-Aragarças, a
$111 \mathrm{~km}$ de Jussara, 11.XI.1999, C. P. Bove et al. 525 (R); estrada Aruanã-Araguapaz, a 22,4 km de Aruanã, 15.XI.1999, C. P. Bove et al. 601 (R).

Distribuição geográfica: América do Norte, América Central, Caribe, América do Sul, Ásia, África, Madagascar e Oceania. No Brasil, a espécie ocorre nos estados de Bahia, Goiás, Mato Grosso, Mato Grosso do Sul, Minas Gerais, Pernambuco, Rio de Janeiro, Rio Grande do Sul, Roraima, Santa Catarina e São Paulo.

7. Eleocharis capillacea Kunth, Enum. Pl. 2: 139. 1837.

Material examinado: BRASIL, Goiás: estrada Jussara-Aruanã, no km 40, 28.V.1997, C. P. Bove et al $199 b(\mathrm{R})$.

Distribuição geográfica: América Central, Caribe e América do Sul. No Brasil, a espécie ocorre nos estados de Bahia, Goiás, Minas Gerais, Paraná, Roraima, Santa Catarina, São Paulo e no Distrito Federal.

8. Eleocharis filiculmis Kunth, Enum. Pl. 2: 144.1837.

Material examinado: BRASIL, Goiás: estrada Jussara-Aragarças, no km 100 (lado esquerdo), 26.V.1997, C. P. Bove et al. 184 (R); estrada Jussara-Aragarças, a $97 \mathrm{~km}$ de Jussara, 11.XI.1999, C. P. Bove et al. 509 (R); estrada Jussara-Britânia (via Jacilândia), a 78,2 km de Jussara, 12.XI.1999, C. P. Bove et al. 544 (R).

Distribuição geográfica: México, América Central, Caribe e América do Sul. No Brasil, a espécie ocorre nos estados de Bahia, Goiás, Mato Grosso, Mato Grosso do Sul, Paraná, Piauí, Pernambuco, Rio Grande do Sul, Roraima, Santa Catarina, São Paulo e Tocantins.

9. Eleocharis interstincta (Vahl) Roem. \& Schult., Syst. Veg. 2: 149. 1817.

Scirpus interstinctus Vahl, Enum. Pl. 2: 251. 1805.

Material examinado: BRASIL, Goiás: estrada Aruanã-Britânia, no km 20, 14.X.1997, C. P. Bove et al. 291 (R); estrada Jussara-Britânia (via Jacilândia), a 58,9 km de Jussara, 12.XI.1999, C. P. Bove et al. 552 (R).

Distribuição geográfica: Estados Unidos, México, América Central, Caribe, América do Sul e África do Sul. No Brasil, a espécie ocorre nos estados de Acre, Bahia, Ceará, Espírito Santo, Goiás, Maranhão, Mato Grosso, Mato Grosso do Sul, Minas Gerais, Pará, Para- 
íba, Paraná, Pernambuco, Rio de Janeiro, Rio Grande do Sul, Roraima, Santa Catarina e São Paulo.

10. Eleocharis minima var. bicolor (Chapm.) Svenson, Rhodora 39: 219.1937.

Eleocharis bicolor Chapm., Fl. South. U. S. 517. 1860.

Material examinado: BRASIL, Goiás: estrada Aruanã-Araguapaz, a 22,4 km de Aruanã, 15.XI.1999, C. P. Bove et al. 592 (R); estrada Jussara-Aruanã, no km 40, 28.V.1997, C. P. Bove et al 199a (R).

Distribuição geográfica: Estados Unidos, México, América Central, Caribe e América do Sul. No Brasil, a espécie ocorre nos estados de Goiás, Santa Catarina e São Paulo.

11. Eleocharis nana Kunth, Enum. Pl. 2: 140. 1837.

Material examinado: BRASIL, Mato Grosso: Santa Cruz, 1892, S. Moore 530 (R).

Distribuição geográfica: Estados Unidos, México, Cuba, Guiana e Uruguai. No Brasil, a espécie ocorre nos estados da Bahia, Mato Grosso, Paraná, Rio de Janeiro, Rio Grande do Sul, Santa Catarina e São Paulo.

12. Eleocharis nudipes (Kunth) Palla, Denkschr. Akad. Wiss. Wien 79: 171. 1908.

Isolepis nudipes Kunth, Enum. Pl. 2: 206. 1837.

Material examinado: BRASIL, Goiás: estrada Jussara-Aragarças, no km 100 (lado esquerdo), 26.V.1997, C. P. Bove et al. 185 (R); estrada Jussara-Aragarças, no km 100 (lado esquerdo), 26.V.1997, C. P. Bove et al. 188 (R); estrada Jussara-Aragarças, a $100 \mathrm{~km}$ de Jussara, 09.X.1997, C. P. Bove et al. 230a (R); estrada Jussara-Britânia (via Jacilândia), a 14,3 km de Jussara, 12.XI.1999, C. P. Bove et al. 568 (R).

Distribuição geográfica: Argentina, Paraguai e Uruguai. No Brasil, a espécie ocorre nos estados de Goiás, Mato Grosso do Sul, Minas Gerais, Paraná, Rio Grande do Sul, Santa Catarina e São Paulo.

13. Eleocharis plicarhachis (Griseb.) Svenson, Rhodora 31: 158. 1929.

Scirpus plicarhachis Griseb., Cat. Pl. Cub. 239. 1866.

Material examinado: BRASIL, Goiás: estrada Jussara-Aragarças, a $100 \mathrm{~km}$ de Jussara, 09.X.1997, C. P. Bove et al. $230 b$ (R).
Distribuição geográfica: sul do México, América Central e América do Sul. No Brasil, a espécie ocorre nos estados de Amazonas, Bahia, Goiás, Mato Grosso, Minas Gerais, Paraíba, Paraná, Roraima, Rio Grande Sul, Santa Catarina e São Paulo.

14. Eleocharis retroflexa (Poir.) Urb., Symb. Antill. 2: 165. 1900.

Scirpus retroflexus Poir., Encycl. 6: 753.1804.

Material examinado: BRASIL, Goiás: estrada Aruanã-Peixe, a $25 \mathrm{~km}$ do trevo, 13.IV.2006, C. P. Bove et al. 1649 (R).

Distribuição geográfica: América Central, Caribe, América do Sul, África e Madagascar. No Brasil, a espécie ocorre nos estados de Bahia, Goiás e Roraima.

15. Eleocharis sellowiana Kunth, Enum. Pl. 2: 149. 1837.

Material examinado: BRASIL, Mato Grosso: Alto Araguaia, Córrego do Sapo, 14.I.2004, C. P. Bove et al. 1326 (R).

Distribuição geográfica: América Central e América do Sul. No Brasil, a espécie ocorre nos estados de Bahia, Ceará, Espírito Santo, Goiás, Maranhão, Mato Grosso, Minas Gerais, Pernambuco, Rio de Janeiro, Rio Grande do Sul, Santa Catarina, São Paulo e no Distrito Federal.

16. Fimbristylis aestivalis (Retz.) Vahl, Enum. Pl. 2: 288. 1805.

Scirpus aestivalis Retz., Observ. Bot. 4: 12. 1791.

Material examinado: BRASIL, Goiás: estrada Mozarlândia-Nova Crixás, a 32,3 km do trevo, 15.XI.1999, C. P. Bove et al. 608a (R).

Distribuição geográfica: América Central, América do Sul. No Brasil, a espécie ocorre nos estados de Goiás, Mato Grosso e Roraima.

17. Fimbristylis dichotoma (L.) Vahl, Enum. Pl. 2: 287. 1805.

Scirpus dichotomus L., Sp. Pl. 1: 50. 1753.

Material examinado: BRASIL, Goiás: estrada Jussara-Aragarças, a $3 \mathrm{~km}$ de Jussara, Rio Água-Limpa, 11.XI.1999, C. P. Bove et al. 486 (R); estrada Jussara-Aragarças, a $97 \mathrm{~km}$ de Jussara, 11.XI.1999, C. P. Bove et al. 510 (R).

Distribuição geográfica: Estados Unidos, América Central, América do Sul, Ásia, África, Madagascar e Austrália. No Brasil, a espécie ocorre nos estados de Acre, Amazo- 
nas, Bahia, Goiás, Mato Grosso do Sul, Minas Gerais, Pará, Paraíba, Pernambuco, Piauí, Rio de Janeiro, Rio Grande do Sul, Roraima e São Paulo.

18. Fimbristylis miliacea (L.) Vahl, Enum. P1. 2: 287. 1805.

Scirpus miliaceus L., Syst. Nat. 10: 868. 1759.

Material examinado: BRASIL. Tocantins: estrada Formoso do Araguaia para São João do Javaés (BR-242), a $31 \mathrm{~km}$ de Formoso do Araguaia, 15.IV.2006, C. P. Bove et al. 1668 (R).

Distribuição geográfica: sul dos Estados Unidos, México, América Central, Caribe, América do Sul, Ásia e África. No Brasil, a espécie ocorre nos estados de Amapá, Amazonas, Mato Grosso do Sul, Pernambuco, Roraima e Tocantins.

19. Fuirena umbellata Rottb., Descr. Icon. Rar. Pl. 70. 1773.

Material examinado: BRASIL, Goiás: estrada Jussara-Britânia (via Jacilândia), a 78,2 km de Jussara, 12.XI.1999, C. P. Bove et al. 541 (R); estrada Jussara-Britânia (via Jacilândia), a 78,2 km de Jussara,12.XI.1999, C. P. Bove et al. 540 (R); estrada Jussara-Aragarças, a 100 km de Jussara, 11.XI.1999, C. P. Bove et al. 522 (R); estrada Jussara-Aragarças, a $111 \mathrm{~km}$ de Jussara, 11.XI.1999, C. P. Bove et al. 528 (R).

Distribuição geográfica: sul do México, América Central, Caribe, América do Sul, África e Madagascar. No Brasil, a espécie ocorre nos estados de Alagoas, Amazonas, Bahia, Ceará, Espírito Santo, Goiás, Mato Grosso do Sul, Minas Gerais, Pará, Paraíba, Paraná, Pernambuco, Rio de Janeiro, Roraima, Santa Catarina e São Paulo.

20. Lipocarpha humboldtiana Ness, Linnaea 9: 287. 1834.

Material examinado: BRASIL, Goiás: estrada Jussara-Britânia (via Jacilândia), a 78,2 km de Jussara, 12.XI.1999, C. P. Bove et al. 548 (R).

Distribuição geográfica: sul do México, América Central e América do Sul. No Brasil, a espécie ocorre nos estados de Bahia, Goiás, Mato Grosso, Minas Gerais, Paraná, Rio Grande do Sul, Roraima, Santa Catarina e São Paulo.

21. Pycreus unioloides (R. Br.) Urb., Symb. Antill. 2(1): 164. 1900. 1810.

Cyperus unioloides R. Br., Prodr. 216.
Material examinado: BRASIL, Goiás: estrada Jussara-Aragarças, a $100 \mathrm{~km}$ de Jussara, 11.XI.1999, C. P. Bove et al. 518 (R).

Distribuição geográfica: América do Norte, América Central, América do Sul, Ásia, África e Austrália. No Brasil, a espécie ocorre nos estados de Goiás, Mato Grosso do Sul, Minas Gerais, Rio Grande do Sul e Santa Catarina.

22. Rhynchospora barbata (Vahl) Kunth., Enum. Pl. 2: 290. 1837.

Schoenus barbatus Vahl, Eclog. Amer. 2: 4. 1798.

Material examinado: BRASIL, Goiás: estrada Aruanã-Araguapaz, no km 4, 29.V.1997, C. P. Bove et al. $218 b$ (R).

Distribuição geográfica: sul do México, América Central, Caribe e América do Sul tropical. No Brasil, a espécie ocorre nos estados de Amapá, Bahia, Ceará, Goiás, Mato Grosso do Sul, Pará, Paraíba, Pernambuco, Piauí, Roraima e Sergipe.

23. Rhynchospora brevirostris Griseb., Cat. Pl. Cub. 246. 1866.

Material examinado: BRASIL, Mato Grosso: rio Manso, III.1916, F. C. Hoehne 6931a (R); a $25 \mathrm{~km}$ ao sul de Xavantina, 13.VI.1966, H. S. Irwin et al. s/n (RB162141).

Distribuição geográfica: América Central, Caribe, América do Sul, África e Madagascar. No Brasil, a espécie ocorre nos estados de Bahia, Goiás, Mato Grosso, Mato Grosso do Sul, Pernambuco e São Paulo.

24. Rhynchospora corymbosa (L.) Britton, Trans. New York Acad. Sci. 11: 84. 1892.

Scirpus corymbosus L., Cent. Pl. 2: 7. 1756.

Material examinado: BRASIL, Goiás: estrada Mozarlândia-Nova Crixás, a $37 \mathrm{~km}$ do trevo, 15.XI.1999, C. P. Bove et al. 614 (R).

Distribuição geográfica: América Central, Caribe, Antilhas, América do Sul, Ásia, África e Madagascar. No Brasil, a espécie ocorre nos estados de Alagoas, Amazonas, Bahia, Ceará, Goiás, Mato Grosso, Mato Grosso do Sul, Minas Gerais, Pará, Paraná, Pernambuco, Rio de Janeiro, Rio Grande do Sul, Roraima, Santa Catarina, São Paulo e no Distrito Federal.

25. Rhynchospora globosa (Kunth) Roem. \& Schult. Syst. Veg. 2: 89. 1817. 
Chaetospora globosa Kunth, Nov. Gen. Sp. 1: 230.1815 (1816).

Material examinado: BRASIL, Goiás: cerca de $12 \mathrm{~km}$ ao sul de Caiapônia, 02.V.1973, W. R. Anderson 9652 (R).

Distribuição geográfica: América Central, Caribe, Antilhas e América do Sul tropical. No Brasil, a espécie ocorre nos estados de Amapá, Amazonas, Bahia, Goiás, Mato Grosso, Mato Grosso do Sul, Minas Gerais, Pará, Paraíba, Paraná, Pernambuco, Piauí, Rio Grande do Sul, Roraima, Santa Catarina, São Paulo e no Distrito Federal.

26. Rhynchospora trispicata (Nees) Schrad ex Steud, Syn. Pl. Glumac. 2: 145. 1855.

Ephippiorhynchium trispicatum Nees, Fl. Bras. 2(1): 136. 1842.

Material examinado: BRASIL, Goiás: estrada Aruanã-Peixe, a $25 \mathrm{~km}$ do trevo, 13.IV.2006, C. P. Bove et al. 1658 (R).

Distribuição geográfica: México, América Central e América do Sul. No Brasil, a espécie ocorre nos estados de Amapá, Amazonas, Goiás, Mato Grosso, Mato Grosso do Sul e Roraima.

27. Rhynchospora velutina (Kunth) Boeck. Vindensk. Meddel. Dansk Naturhist. Foren. Kjobenhavn 31: 149. 1869.

Dichromena velutina Kunth, Enum. Pl. 2: 282. 1837.

Material examinado: BRASIL, Goiás: Serra dos Caiapós, a $40 \mathrm{~km}$ de Amarinópolis para Rio Verde, 20.III.1971, J. A. Rizzo 6106 (UFG); Serra dos Caiapós, a 40 km de Amarinópolis para Rio Verde, 17.IV.1971, J. A. Rizzo 6220 (UFG).

Distribuição geográfica: México, América Central e América do Sul. No Brasil, a espécie ocorre nos estados de Bahia, Goiás, Mato Grosso, Mato Grosso do Sul, Paraíba, Paraná, Pernambuco, Rio Grande do Sul, Roraima, São Paulo e no Distrito Federal.

28. Scleria melaleuca Rchb. ex Schltdl. \& Cham, Linnaea 6(1): 29. 1831.

Material examinado: BRASIL, Goiás: estrada Aruanã-Canga, a $25 \mathrm{~km}$ do trevo, 13.IV.2006, C. P. Bove et al. 1653 (R).

Distribuição geográfica: sul do México, América Central, Caribe, Antilhas, América do Sul, África tropical e Madagascar. No Bra- sil, a espécie ocorre nos estados de Amazonas, Bahia, Espírito Santo, Goiás, Maranhão, Minas Gerais, Pará, Paraíba, Pernambuco, Rio de Janeiro, São Paulo, Tocantins e no Distrito Federal.

29. Scleria mitis P. J. Bergius, Kongl.Vetensk. Acad. Handl. 26: 145. 1765.

Material examinado: BRASIL, Goiás: estrada Jussara-Britânia (via Jacilândia), a $25 \mathrm{~km}$ de Jussara, 12.XI.1999, C. P. Bove et al. 564 (R).

Distribuição geográfica: América Central, Caribe, Antilhas e América do Sul. No Brasil, a espécie ocorre nos estados de Acre, Bahia, Goiás, Mato Grosso, Mato Grosso do Sul, Minas Gerais, Pernambuco, Roraima, São Paulo e no Distrito Federal.

\section{Agradecimentos}

À Universidade Federal do Estado do Rio de Janeiro/UNIRIO, pela bolsa de aperfeiçoamento concedida ao primeiro autor; ao $\mathrm{CNPq}$, pela bolsa de mestrado concedida ao segundo autor; ao Programa de Pós-graduação em Ciências Biológicas (Botânica) - Museu Nacional/UFRJ, pela utilização das instalações e infra-estrutura cedidas durante a realização do presente trabalho; ao Dr. José Ângelo Rizzo, da Universidade Federal de Goiás / UFG, e aos funcionários dos herbários R, RB, UEC e UFG, pela ajuda e disponibilidade.

\section{REFERÊnCIAS}

Adams, C. D. 1994. Cyperaceae, p. 402-485. In: G. Davidse, M. Souza \& A. Charter (Eds), Flora mesoamericana 6. Cidade do México, Universidad Nacional Autónoma de México.

Barros, M. 1960. Las Ciperáceas del Estado de Santa Catarina. Sellowia 12: 181-450.

Bove, C. P., A. S. B. Gil, C. B. Moreira \& R. F. B. Anjos. 2003. Hidrófitas fanerogâmicas de ecossistemas aquáticos temporários da planície costeira do Estado do Rio de Janeiro, Brasil. Acta Bot. Bras. 17: 119-135.

Bruhl, J. J. 1995. Sedge genera of the world: relationships and a new classification of the Cyperaceae. Austral. Syst. Bot. 8: 125-305. 
Diego-Pérez, N. 1997. Cyperaceae, p.1-169. In: N. Diego-Pérez \& R. M. Fonseca (Eds), Flora de Gerrero 5. Cidade do México, Universidad Nacional Autónoma de México.

Dubs, B. 1998. Prodromus florae matogrossensis. The botany of Mato Grosso. Betrona-Verlag, Küsnacht, 444 p.

Esteves, F. A. 1988. Fundamentos de limnologia. Interciência, Rio de Janeiro, 575 p.

Faria, A.D. 1998. O gênero Eleocharis R. Br. (Cyperaceae) no Estado de São Paulo. Dissertação de Mestrado, Universidade Estadual de Campinas, Campinas.

Gil, A.S.B. \& C.P. Bove. 2004. O gênero Eleocharis R. Br. (Cyperaceae) nos ecossistemas aquáticos temporários da planície costeira do Estado do Rio de Janeiro. Arq. Mus. Nac. 62: 131-150.

Gil, A.S.B. \& C.P. Bove. 2007. Eleocharis R. Br. (Cyperaceae) no estado do Rio de Janeiro, Brasil. Biota Neotróp. 7: 1-31.

Goetghebeur, P. 1998. Cyperaceae, p. 141-190. In: K. Kubitzki (Ed), The families and genera of vascular plant. IV. Flowering plants-monocotyledons. Berlin, SpringerVerlag.

Guaglianone, E. R. 2001. Contribution to the study of the genus Rhynchospora (Cyperaceae) V. Section Longirostres in Austral America. Darwiniana 39: 287-342.

Guaglianone, E. R. \& G. Moore. 2001. Identity of two South American species of Rhynchospora (Cyperaceae): $R$. rostrata and $R$. organensis. Darwiniana 39: 239-246.

Hoehne, F.C. 1948. Plantas aquáticas. Secretaria de Agricultura do Estado de São Paulo, São Paulo, 168 p.

IBGE. 1989. Geografia do Brasil - região Centro-Oeste. v.1. Rio de Janeiro, 92 p.

Irgang, B. E. \& C. V. S. Gastal Jr. 1996. Macrófitas aquáticas da planície costeira do RS. Ed. Porto Alegre, Porto Alegre, 290 p.

Jorgensen, P. M. \& C. Ulloa. 1994. Seed plants of the High Andes of Ecuador - a checklist. AAU Rep. 34: 182-188.

Judd, W. S., C. S. Campbell, E. A. Kellogg, P. F. Stevens \& M. J. Donoghue. 2002. Plant systematic: a phylogenetic approach. 2nd ed. Sinaver Associates Inc., Sunderland, $576 \mathrm{p}$.
Kerns, D. M., W. W. Thomas, G. C. Tucker, R. Kral, K. Camelbeke, D. A. Simpson, A. A. Reznicek, M. S. González-Elizondo, M. T. Strong \& P. Goetghebeur. 1998. Cyperaceae. p. 486-663. In: J. A. Steyermark, P. E. Berry \& B. K. Holst (Eds), Flora of the Venezuelan Guayana. St. Louis, Missouri Botanical Garden Press.

Koehler, S. \& C.P. Bove. 2001. Hydrocharitaceae from central Brazil: a new species of Egeria and a note on Apalanthe granatensis. Novon 11: 63-66.

Koehler, S. \& C.P. Bove. 2004. Alismatales from the upper and middle Araguaia river basin (Brazil). Rev. Brasil. Bot. 27: 439-452.

Kuhlmann, M. \& E. Kühn. 1947. A flora do Distrito de Ibiti (ex - Monte Alegre), Município de Amparo. Instituto de Botânica, São Paulo, 221 p.

Lawesson, J. E., H. Adsersen \& P. Bentley. 1987. An updated and annotated checklist of the vascular plants of the Galapagos Islands. Rep. Bot. Inst. Univ. AARHUS 16: 67-69.

Luceño, M., M.V. Alves \& A.P. Mendes. 1997. Catálogo florístico y claves de identificación de las Ciperáceas de los Estados de Paraíba y Pernambuco (Nordeste do Brasil). Anal. Jard. Bot. Madrid 55: 67-100.

Marticorena, C. \& M. Quezada. 1985. Catálogo de la flora vascular de Chile. Gayana Bot. 42: 1-157.

Martins, M. L. L., R. M. Carvalho-Okano \& M. Luceño. 1999. Cyperaceae do Parque Estadual Paulo César Vinha, Guarapari, Espírito Santo, Brasil. Acta Bot. Bras. 13: 187-222.

Matias, L. Q., E. R. Amado \& E. P. Nunes. 2003. Macrófitas aquáticas da Lagoa de Jijoca de Jericoacoara, Ceará, Brasil. Acta Bot. Bras. 17: 623-631.

Mori, S. A., L. A. M. Silva, G. Lisboa \& L. Coradin. 1989. Manual de manejo de herbário fanerogâmico. Centro de Pesquisas do Cacau, Ilhéus, $104 \mathrm{p}$.

Muenscher, W. C. 1944. Cyperaceae, p. 141174. In: W. C. Muenscher (Ed), Aquatic plants of the United States. New York, Comstock Publishing Company Inc. 
Muniz, C. F. S. 2001. Cyperaceae, p. 60-63. In: A. F. Costa \& I. C. A. Dias (Orgs), Flora do Parque Nacional da Restinga de Jurubatiba e arredores, Rio de Janeiro, Brasil: listagem florística e fitogeografia: Angiospermas, Pteridófitas, Algas continentais. Rio de Janeiro, Editora do Museu Nacional do Rio de Janeiro.

Muniz, C. F. S. \& G. Shepherd. 1987. O gênero Scleria Berg. (Cyperaceae) no estado de São Paulo. Rev. Brasil. Bot. 10: 63-94.

Nees, C. G. 1842. Cyperaceae, p. 1-226. In: C. F. P. Martius (Ed), Flora Brasiliensis, v. 2, pars 1. Lipsiae, F. Fleischer.

Nelson, C. 1978. Contribuciones a la flora de la Mosquitia, Honduras. Ceiba 22: 41-64.

Oliveira, E. C. 1980. Cyperaceae Juss. morfologia dos aquênios de gêneros ocorrentes no Brasil. Rodriguésia 32: 327-405.

Pedralli, G., J. R. Stehmann, M. C. B. Teixeira, V. L. Oliveira \& S. T. Meyer. 1993. Levantamento da vegetação aquática (macrófitas) na área da EPDA-Peti, Santa Bárbara, MG. Iheringia, Sér. Bot. 43: 1528.

Pott, V. J. \& Pott, A. 1997. Checklist das macrófitas aquáticas do Pantanal, Brasil. Mato Grosso do Sul. Acta Bot. Bras. 11: 215-227.

Prata, A.P. 2002. Listagem florística das Cyperaceae do Estado de Roraima, Brasil. Hoehnea 29: 93-107.

Renner, S. S., H. Balslev \& L. B. Holm-Nielsen. 1990. Flowering plants of Amazonian Ecuador - a checklist. AAU Rep. 24: 8890.

Rocha, E. A. \& M. Luceño. 2002. Estudo taxonômico de Rhynchospora Vahl seção Tenues (Cyperaceae) no Brasil. Hoehnea 29: 189-214.

Rosa, F. F. \& B. E. Irgang. 1998. Comunidades vegetais de um segmento da planície de inundação do Rio dos Sinos, Rio Grande do Sul, Brasil. Iheringia, Sér. Bot. 50: 7587.

Sampaio, A. J. 1916. A flora do Mato Grosso. Arq. Mus. Nac. Rio de Janeiro, 19: 1-125.
Schessl, M. 1999. Floristic composition and structure of floodplain vegetation in the Northern pantanal of Mato Grosso. Brazil. Phyton 39: 303-336.

Simpson, D. A. 1995. Cyperaceae, p. 661-683. In: B. Stannard (Ed), Flora of the Pico das Almas. Kew, Royal Botanic Gardens.

Soltis, D. E., P. S. Soltis, P. K. Endress \& M. W. Chase. 2005. Phylogeny and evolution of Angiosperms. Sinaver Associates Inc., Sunderland, $370 \mathrm{p}$.

Standley, P. C. \& J. A. Steyermark. 1958. Cyperaceae. Fieldiana Bot. 24: 90-196.

Svenson, H. K. 1929. Monographic studies in the genus Eleocharis. Rhodora 31:121-135, 152-163, 167-191, 199-219, 224-242.

Svenson, H. K. 1932. Monographic studies in the genus Eleocharis. Rhodora 34:193-203, 215-227.

Svenson, H. K. 1934. Monographic studies in the genus Eleocharis. Rhodora 36:377389.

Svenson, H. K. 1937. Monographic studies in the genus Eleocharis. Rhodora 39:210-231, 236-273.

Svenson, H. K. 1939. Monographic studies in the genus Eleocharis. Rhodora 41:1-19, 4377, 90-110.

Thomas, W. W. 1996. Notes on capitate Venezuelan Rhynchospora (Cyperaceae). Brittonia 48: 481-486.

Trevisan, R. 2005. O gênero Eleocharis R. Br. (Cyperaceae) no Rio Grande do Sul. Dissertação de Mestrado, Universidade Federal do Rio Grande do Sul, Porto Alegre.

Tropicos.org. Missouri Botanical Garden. Disponível em: <http://www. tropicos.org>. Acesso em 8 jan. 2007.

Tundisi, J.G. \& F.A.R. Barbosa. 1995. Conservation of aquatic ecossystems: present status and perspectives, p. 365-376. In: J.G. Tundisi, C.E.M. Bicudo \& T. Matsumura (Eds), Limnology in Brazil. Rio de Janeiro, Associação Brasileira de Ciências/Sociedade Brasileira de Limnologia.

Velasquez, J. 1994. Plantas acuáticas vasculares de Venezuela. Universidad Central de Venezuela, Caracas, 992 p.

Recebido em 26/III/2007 Aceito em $21 / \mathrm{X} / 2008$ 